\title{
The first massive astronomical observation event in Mexico City
}

\section{Mariana Espinosa and Xavier Hernandez}

\author{
Instituto de Astronomía, \\ Universidad Nacional Autónoma de México, \\ UNAM A.P. 70264, C.P. 04510 México D.F., México \\ email: mespinosa@astroscu.unam.mx xavier@astroscu.unam.mx
}

\begin{abstract}
On the night of the 20th of February 2008 there was a total eclipse of the moon visible from Mexico City, with a total duration from 19:42 hrs to 23:09 hrs. At the Instituto de Astronomía, UNAM, we took this opportunity to organise a massive astronomical party on the central plaza of the city, the Zocalo. Over a period of about $6 \mathrm{hrs}$. we set up a huge astro-party, with free use of over 100 telescopes, where we estimate over 40,000 persons looked through an astronomical telescope at the moon and Saturn, most for the first time in their lives. Numerous stands including a children's games, an Astronomy conference room, and the free distribution of Astronomical material were organised. Here we describe some of the issues associated with the planning and implementation of the event. Coordination issues were complex, involving interaction with divers and numerous authorities, city, national, police, traffic, medical assistance in readiness, aide from other universities, and amateur astronomical societies, which supplied most of the telescopes. An extensive publicity campaign was launched with several weeks of anticipation, and although we had no way of estimating the public response, we were ready with over 800 volunteers at the Zócalo on the 20th of February. The public response was massive and overwhelmingly positive, thousands swarmed the square in a completely peaceful and well organised interaction between Astronomy and society at large, over many complementary levels
\end{abstract}

Keywords. Public outreach, astronomical events, Mexico City

\section{Motivation}

The central square in Mexico City, called the Zócalo, is a traditional focal point for cultural and political life in the city. There exists a long tradition of massive events at the place, with the local population being well accustomed to meetings of large numbers at the Zócalo, for a wide variety of purposes. Never before however, had science been the topic of a large scale reunion there. The lunar eclipse of the 20th of February 2008 offered a unique opportunity to harness the potential of the Zócalo for astronomy. Headed by the communication office of the Instituto de Astronomía, UNAM, where all the planning and general coordination of the event took place, we prepared a massive star party. This was to be centred on the lunar eclipse, with several important goals in mind.

The recovery of the joy of the night sky was central. As with all large cities, light pollution in Mexico City has reduced the night sky to an orange haze above the millions of artificial light, occasionally marked by a handful of stars and the moon. Although the large scale lighting environment of Mexico City night was a background with we were forced to adapt to, locally, the relative hight rectangular official buildings and not overly wide streets defining the colonial square, offered the chance to create a darkened box relatively shielded off from most direct light sources, if we managed to have all lighting within the square turned off. 
The bright nature of the lunar eclipse made it ideal for the viewing conditions, providing an accessible astronomical event, ordinarily lost to the general public. Astronomical observations with small and medium telescopes were also planned, almost exclusively to observe Saturn, which appeared fairly bright at the time. It was our intention to highlight some of the richness of the night sky, and to focus attention on how light pollution has practically erased an important feature of the natural world for city dwellers.

The opportunity was also seen as a chance to rehearse for the type of events and large scale coordination which at the Instituto de Astronomia we had started to plan for the 2009 International Year of Astronomy, while the extent of the publicity surrounding the event allowed the astronomical community to prime the public on the coming IYA2009. also, it offered an excuse to set in motion all the various collaborative efforts and inter-institutional links which will be required to make the IYA2009 as successful as possible. Contacts with both city and national cultural authorities were initiated, as well as large scale coordination between various astronomical institutions participating, UNAM, INAOE, IPN and others. Of fundamental importance were the contacts and coordination efforts undertaken with the amateur astronomical community, which furnished the overwhelming majority of the astronomical telescopes offered to the public that day, over 100 .

Further, it gave the astronomical community in Mexico a chance to practice and refine the type of events we could be capable of pulling off, to gauge the extent of our possibilities, as well as some empirical experience on what could go wrong, and hence how to better plan for IYA2009 events. We fortunately succeeded in counting with the good will and cooperation of 14 amateur astronomical societies from various states of Mexico, the continual help of which will be instrumental for the optimal realization of various goals of the IYA2009 in Mexico.

Regarding science communication, the goal was to give thousands of people first hand, direct awareness of the phenomenon of eclipses, both the visual appearance and temporal development, as well as scientific notions of the causes and explanation of the event. Bringing people into contact with the science of astronomy was also important, both the observational aspect, through access to astronomical telescopes, and also the wider undertaking of the science of Astronomy. Towards this aim, a small auditorium was installed where "Chat with the Astronomers" sessions were organised. There, a number of professional astronomers devoted long hours to answering an incredible variety of questions from the public, and to the telling of their professional lives.

\section{Planning}

Planning started some 4 months in advance, a period which proved barely sufficient. After putting the project together, the first point was to sell the idea to the city authorities, not only was their permission explicitly needed, but without their enthusiastic cooperation, the event would have been impossible. We were lucky in securing fairly rapidly the approval and partnership of the local authorities, the Gobierno de la Ciudad de Mexico, leading to help from the education and science offices of said government. Other higher education and research institutions joined enthusiastically as soon as the project was perceived as active and running smoothly. The main institutions involved were: Instituto de Astronomía and Science Museum "Universum" both part of the Universidad Nacional Autónoma de Mexico (UNAM), Instituto Nacional de Astrofísica Optica y Electrónica (INAOE), Instituto Politecnico Nacional and fourteen amateur groups from the centre area of the country such as Nibiru and Safir, both associated to the Schools of Science and 
Engineering at UNAM. Their addresses can be found at the Mexican IYA2009 webpage http://www . astronomia2009.org.mx.

After some hesitation surrounding the idea of plunging into darkness the seat of the Mexico City government, with thousands gathered in the square, the go ahead was obtained from the city authorities for as full a blackout as possible. The National palace, formal seat of the national government of Mexico, lines one of the sides of the Zócalo. Turning off lights at the National Palace, required authorisation from the federal national government, not always on good terms with the local city authorities. Luckily, this too was secured, as was the cooperation of the traffic authorities, which agreed to shutting off the traffic and traffic lights of the roads circling the central square, as well as of the roads leading into it, for 1 block each. Including the roads, we were left with a darkened square with close to 300 metres on a side.

The climate in Mexico city is characterised by a rainy season in summer, and fairly dry weather during winter. Still, February is known for its 'crazy' weather, with unpredictable rains occasionally taking place. We counted on clear skies, but some sort of a back up was in order, in case rain or clouds could spoil the astronomical observations. It was suggested that installing large screens to project astronomical images and film could be an answer, to allow us to provide to the crowd, should the sky be overcast. This was somewhat controversial, as it appeared to be inconsistent with the light pollution awareness theme, the screens could potentially spoil the astronomical observations themselves. In the end we arranged for 2 large $5 \mathrm{mx} 3 \mathrm{~m}$ screens, where astronomical images and live transmission of the eclipse from 2 astrophysical observatories in Mexico could be shown, mostly as a back up plan. Care was also taken to ensure the orientation and placing of the screens did only minimally affect the telescope observations.

\section{The event}

At the beginning of the evening there was a performance by a modern ballet group, followed by a public lecture on Galileo that included hands-on demonstrations of elementary physic. With the twilight of the setting sun a mambo group danced the première of "and yet it moves", a mambo composed specifically for the event, and performed by a full orchestra.

There was an official ceremony with the Mayor of Mexico City, the Presidents of the UNAM and of the Instituto Politécnico Nacional, as well as the secretaries of Science and Education of the City. In exchange for the science theme photo opportunity, we got substantial support and formal backing for the event, essentially a pre-launch of the IYA2009 activities in Mexico.

A large stand with children's activities centred on astronomical themes was installed, contributing to making the event an all family affair. Telescope workshops were set up, where amateur astronomer associations publicised their activities, and attracted people to the public viewing sessions. A set of large astronomy posters with explanations on the lunar eclipse and Saturn were placed in the fashion of a science exhibit, at the far end of the plaza under very discrete lighting, and with the attendance of graduate students from the Instituto de Astronomía (UNAM), ready to interact with the public and further any explanation or curiosity.

The attendance was estimated to be 20,000 at any one time, altogether the total attendance was about double this figure. Inspite of having over 100 telescopes and very well organized viewing stations, the large turnout resulted in queues of about 1 hour. The observation time per person at the telescopes was about $1 / 2$ minute, with people passing in family groups and getting the chance to look through 2 telescopes, one showing 
Table 1. Statistics of the event

\begin{tabular}{lc}
\hline Public participation & 40,000 \\
Available telescopes & 120 \\
Participating institutions & 14 \\
Amateur astronomical societies & 14 \\
\hline Event staff & \\
Professional iastronomers & 40 \\
Amateur iastronomers & 263 \\
Paramedics and policemen & 40 \\
Students & 70 \\
Artists & 94 \\
Press personnel & 156 \\
\hline Science communication setup & 15,000 \\
Eclipse and night sky flyers & 40 \\
Large astronomy Piposters & 25 \\
Pointing lasers & 5,000 \\
Science magazines & 2 \\
\hline Publicity & 500 \\
Purpose filmed television spots & \\
Posters on public squares and public transport & \\
Various newspaper insertions over a 1.5 month period & \\
Various radio announcements over a 1.5 month period & \\
\hline
\end{tabular}

the Moon, and one Saturn. In order to make the wait more pleasant and interesting, professional astronomers equipped with high power green lasers where constantly walking through the queues, stopping to give short talks on astronomy, and illustrating features of the night sky with the lasers. Overall people where delighted, walking through the large viewing stations one could frequently hear the thrilled expressions of many children and adults on their first direct look at the rings of Saturn or the mountains of the Moon. On leaving the plaza visitors could take flyers with a clear, simple and well illustrated explanation of eclipses on one side, and a map of the winter sky on the other, all 50,000 copies of which were distributed.

Also, we undertook an approximate census of the quantity and quality of the instruments currently owned by Mexican astronomers, both professional and amateur. An extensive promotion of the activities of amateur astronomical associations was achieved, which has resulted in a noticeable increase in the membership of them.

The Eclipse at the Zócalo was a historical and transcendental event. It was replicated in multiple places throughout the country, at locations such as Ensenada, Monterrey, Morelia, Sonora and Puebla. It left an important precedent for science on the streets activities and the popularisation of astronomy through direct experience. It allowed to establish relations amongst local institutions and amateur astronomers who have participated continuously and enthusiastically to this date in the organisation of the IYA2009 activities. In particular, the participation of all those involved in the lunar eclipse event was vital for the organisation of the "Noche de las Estrellas", a similar event where star parties were organised, this time at a national level, at over 20 archaeological and historical sites throughout Mexico. Following the tried and tested scheme, the "Noche de las Estrellas" took place on the 31st of January 2001, and counted with the participation of over 200,000 observers nationwide across Mexico, it constituted a very successful inaugural event for the IYA2009 in Mexico. 